\title{
Characterization and Modeling of Rotational Responses for an Oscillating Foil Underwater Robot
}

\author{
Philippe Giguere \\ Centre for Intelligent Machine \\ McGill University \\ Montreal, Canada \\ philg@cim.mcgill.ca
}

\author{
Chris Prahacs \\ Centre for Intelligent Machine \\ McGill University \\ Montreal, Canada \\ cprahacs@cim.mcgill.ca
}

\author{
Gregory Dudek \\ Centre for Intelligent Machine \\ McGill University \\ Montreal, Canada \\ dudek@cim.mcgill.ca
}

\begin{abstract}
In order to better understand the behavior of the underwater robot developed at our laboratory, a simple but relatively good model of the underwater behavior of the robot had to be developed. In order to be useful for model-based control techniques onboard the robot, the model had to have low computing requirements, yet be complex enough to capture the transient response of the robot. To achieve this, a system identification approach was taken by first capturing the robot response to various inputs, and then matching them to a simple model.
\end{abstract}

Index Terms - Underwater Robotics, Oscillating Foil, Control, Modeling.

\section{INTRODUCTION}

$\mathrm{T}$ his paper examines the development of a simple twolevel top-down model for modeling the behavior of a complex robotic system. We are interested in forming a predictive model for the dynamics of an underwater swimming robot. While analytic models of complex robots have many attractive characteristics, matching them to a physical system is a substantial challenge; this is doubly true in the underwater environment, where faithful hydrodynamic models are very elusive (note this is true for air vehicles as well, and hence wind tunnels remain a staple of the modeling community). In this paper, we develop a simple top-down model based on empirical data and show that this type of "layered" model can be effective even for very complex systems such as our swimmer which uses movable vanes (foils) and which exhibits complex hydrodynamics.

The use of foil-based propulsion systems is gaining greater and greater acceptance for autonomous underwater vehicles (AUVs). A major factor motivating this interest is the highimpulse force and rapid response time these systems are capable of achieving (as well as possible biomimetic properties). Robotuna is one of the first systems employing foil-flapping propulsion, and is a result of pioneering work by Triantafyllou et al. [7]. Its actuated tail is used to generate thrust, propelling the fish-like robot forward. A propulsion system more similar to that being used in our work is the Oscillating Fin Thrusters (OFT) system, which has been developed by Nekton Research [8]. Kemp et al. studied the response time of OFTs [9] and concluded that they showed excellent impulsive forces and response time. A prototype called PilotFish was built to demonstrate the high maneuverability attainable with the use of OFTs [10].

In order to design a robust controller strategy for an AUV, its hydrodynamic characteristics must be studied. Methods used to determine the characteristics fall broadly into two categories: predictive methods and test-based methods. Predictive methods are used when an estimation of system performance is desired prior to completion of the design of the robot, and depend on the careful development of analytical equations, Computational Fluid Dynamics modeling, or on past empirical solutions. On the other hand, test-based methods rely on experimentation to determine the hydrodynamic characteristics of the system. They can therefore only take place once a physical platform is available for testing. Since in our case the robot platform was already operational, the latter method was employed, through the use of the system identification technique [2]. Even though this method is resource intensive (since pool trials are required to collect the data), it comes with a near-guarantee that the model will be close to the real dynamics of the robot. It also avoids the difficulties inherent in estimating all the relevant parameters in predictive methods, particularly in the case of flexible surfaces where the flow dynamics are complex.

The use of a simple model to describe the behavior of the robot has a few key motivating factors:

- If the model is linear, linear control theory techniques such as poles and zeros localization, and estimation of transfer function can be used;

- Model-based control techniques can be readily implemented;

- Model validation is significantly simplified;

- Reinforcement learning with Function Approximation techniques can be used with a greater chance of success.

In prior work [1] a dynamic model for underwater vehicles was presented. The model is divided into basic elements such as the hull, individual control surfaces and propulsion system. Based on that work, a predictive simulator was developed in [3] to help develop and evaluate the swimming gaits for the AQUA robot presented in this paper.

\section{AQUA SWIMMING ROBOT OVERVIEW}

A. AQUA Design Overview 
The robot studied in this paper, referred to as AQUA, is the direct descendant of RHex, a highly mobile six-legged platform [4]. The watertight aluminum shell is rated to depth of $10 \mathrm{~m}$. The robot can swim, walk, maintain station, and crawl at the bottom of the sea using six paddles (flippers). Using these six flippers, the robot can directly control five of its six degrees of freedom.

Although the AQUA design is not optimal as a swimming device, its use of the RHex morphology makes it inherently capable of high-mobility amphibious behaviors such as: swimming, beach landing and walking, stair climbing, etc.

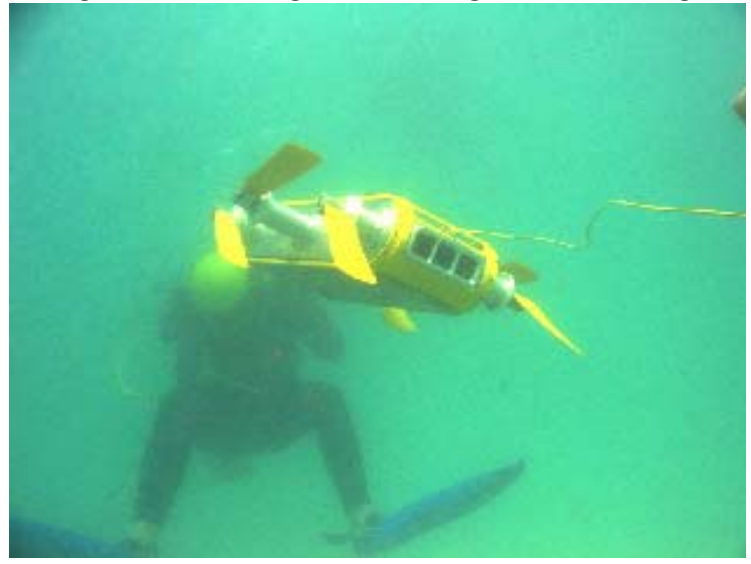

Fig. 1. The AQUA underwater robot in action in Barbados. The six flippers are used to provide both thrust and control. A fiber-optic tether is used to relay visual information and user input commands between the operator control unit and the robot.

\section{B. Swimming Gait and Attitude Control}

The AQUA robot can execute a range of swimming gaits. For the purpose of this experiment, only one swimming gait (called "middle-offset") was employed. In this gait, the flippers oscillate using a sine-function (1), with the middle flippers offset by a phase $\phi=\pi$ relative to the others. This gait produces little body pitch oscillation.

$$
\theta(t)=A \sin (\omega t+\phi)+\theta_{\text {offset }}
$$

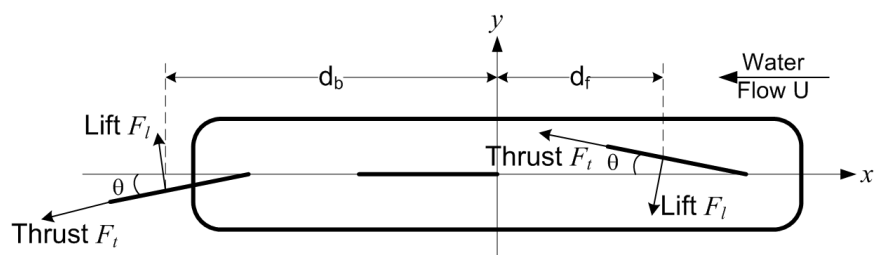

Fig. 2. Force diagram of the robot for a pitch down command. The front flippers are oriented up and the back flippers down.

Pitch and roll moments used to orient the robot are generated by changing the surface oscillation offset angle $\theta_{\text {offset }}$. A pitch command is executed by having $\theta_{\text {offset }}$ set to a value with opposite signs for the front and rear flippers, resulting in a pure pitching moment. A roll command is executed by having $\theta_{\text {offset }}$ set in a similar manner from left to right. The yaw command is executed by creating a net thrust differential between the left and right sides. The thrust differential is obtained by increasing the oscillation amplitude $A$ on one side, and decreasing it on the opposite side.

To avoid abrupt changes in flipper positions, all commands are filtered with a low pass first-order Infinite Impulse Response (IIR) filter, using the discrete-time equation (2), with a time-constant $\tau$ (sampling period $\mathrm{T}=0.001 \mathrm{~s}$ ), where $x$ is the input, and $y$ represents the filtered output. The value $\tau=0.3$ $\mathrm{s}$ has been selected to offer the fastest possible response while keeping commanded motor torques within manufacturer's specifications.

$$
y(n)=\left(1-e^{-\frac{0.001}{\tau}}\right)(x(n)-y(n-1))+y(n-1)
$$

\section{CHARACTERIZATION OF ROTATIONAL RESPONSE}

Although the robot is capable of motion in 6 degrees of freedom, we will restrict this study to the 3 rotational axes (pitch, roll and yaw) given the current sensing capabilities.
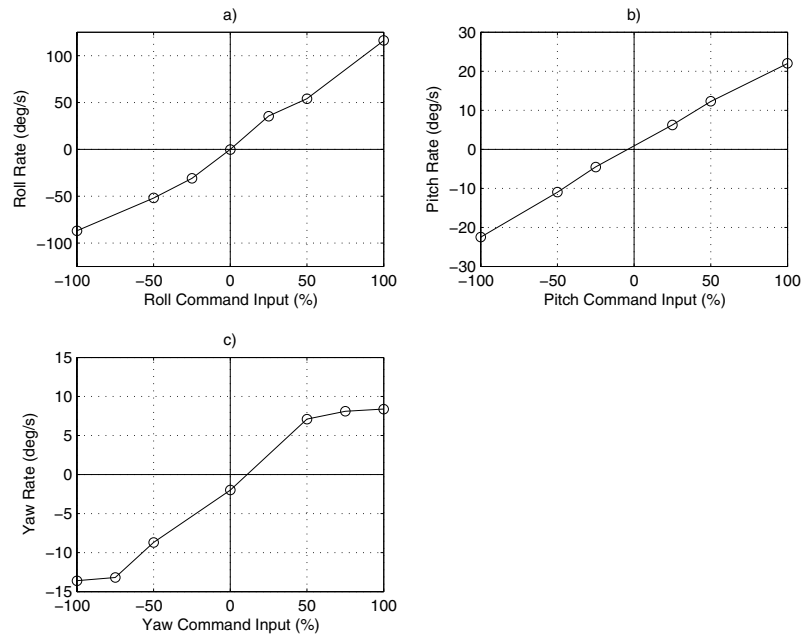

Fig. 3. Steady state response of the robot for the a) roll, b) pitch, and c) yaw axis for single-axis commands. The roll and pitch responses are fairly linear, whereas the yaw shows some saturation past $50 \%$.

The test cases were executed in an indoor pool to minimize external disturbances such as currents on the vehicle. The robot's rotational responses were captured using a Microstrain 3DM-GX1 Inertial Measurement Unit (IMU). This unit provides 3-axis data for each of: angles, accelerations, angular velocities and magnetic fields. Several single-axis test maneuvers were executed in the pool in order to cover the range of possible steering commands for the robot. These maneuvers represented $25 \%, 50 \%$ and $100 \%$ of the full possible command.

Fig. 3 shows the measured rotation rate of the vehicle. One striking feature is the relatively linear relationship between the input command and the angular velocity for pitch and roll axis. The robot shows extremely high maneuverability in the roll axis, with maximum rates exceeding $90 \%$ and response time around $200 \mathrm{~ms}$. The pitch axis maximum rate was lower and stood at $20 \%$, but with still an excellent response time $(250 \mathrm{~ms})$. The response times correspond to the time for the robot to settle to within $10 \%$ of the steady-state rate. 
TABLE I

VEHICLE RESPONSE TIME

\begin{tabular}{cc}
\hline \hline Axis & Response Time \\
\hline Pitch & $200 \mathrm{~ms}$ \\
Roll & $250 \mathrm{~ms}$ \\
Yaw & $1300 \mathrm{~ms}$ \\
\hline \hline
\end{tabular}

The yaw axis response however is by far slower than the previous ones. The maximum rate reached was approximately $12 \%$, for an average response time of $1300 \mathrm{~ms}$, well over the pitch and roll response times. Saturation also appears for yaw commands over $75 \%$. This saturation appears to come from the less-than-optimal thrust characteristics of the flippers at amplitudes greater than $50^{\circ}$, which is $\sim 1.7$ times the nominal amplitude of $30^{\circ}$. Of note is the observed presence of an offset in the yaw rate response, which is thought to arise from small variation in individual flipper construction and bending behavior. This results in non-symmetric thrust characteristics.

\section{Oscillating SuRface Hydrodynamic Forces}

The forces generated by the oscillating flippers can be divided into three components: 1) oscillation-generated thrust, 2) lift and 3 ) drag. The thrust $F_{t}$ generated by the oscillating flippers comes from the resultant flow pattern known as a reverse Karman street, and is assumed to have constant magnitude and oriented at angle $\theta$. The lift $L$ generated by a surface of chord length $D$, at angle $\theta$ with a speed $U$ in a fluid of density $\rho$ can be estimated as shown in [5].

$$
L=\pi \rho D U^{2} \sin \theta
$$

Moreover, some transient lift will be generated by the pitching surface as $\theta$ changes. This force will be proportional to the square of the angular velocity and oriented perpendicular to the angle $\theta$, in the opposite direction to the motion of the flipper surface when seen from the side.

$$
L_{t r}=K_{b} \dot{\theta}^{2}
$$

The total lift generated by the surface will be the sum of (3) and (4):

$$
F_{l}=L+L_{t r}=K_{a} \sin \theta+K_{b} \dot{\theta}^{2}
$$

Although some drag will be generated by the surface parallel to the body length, in this model we will neglect its influence on the vehicle speed $U$.

\section{DYNAMICS MODELING}

To simplify the vehicle model, the following approximations are made:

1) The robot forward speed $U$ remains constant during the maneuvers. This simplifies (3) by making the function only dependant on $\theta$. Although this approximation is clearly violated for large or long lasting commands, its impact can still be somewhat neglected. The speed reduction comes from an increased drag and reduced thrust along the longitudinal axis.
2) Damping force on the vehicle is proportional to angular velocity. This justification will eventually be relaxed by choosing a different damping constant for each command input, since each command generates a different angular velocity of the robot body.

3) The angles $\theta_{\text {offset }}$ for the commands are small. This leads to the common approximations $\sin (\theta)=\theta$ and $\cos (\theta)=1$.

4) The oscillating flippers are modeled as a combination of oriented thrusters and a hydroplane. This approximation is valid as long as the commands are non-periodic, in which case they would interfere with the thrust-generating oscillation.

5) All axes are decoupled. It was experimentally assessed that the cross-talk between the axes is below an acceptable level.

6) The center of buoyancy is the same as the center of gravity. This eliminates one term in the subsequently described Euler motion equation.

The modeling of the robot can be divided into two parts:

1) modeling of the torque generated by the flippers,

2) modeling of the robot response.

\section{A. Body Response Modeling}

The robot response will be modeled in the first place by the Euler equation:

$$
I \ddot{\varphi}+C \dot{\varphi}=M(t)
$$

where $\varphi$ is the robot angle for a given axis, $I$ the moment of inertia of the robot, $C$ the damping and $M(t)$ is the torque applied by the flippers.

\section{B. Torque Generation Modeling}

Fig. 2 shows the force diagram for a pitch command input. The moment $\mathrm{M}$ generated by a flipper will be a combination of the lift generated by the flipper and the orientation of the thrust vector. The pitching moment generated will be the cross product of the moment arm vector and oriented force vector. For small angles, we only consider the forces in the ' $y$ ' direction and assume the moment-arm constant. With the moment arms $d_{f}$ and $d_{b}$ for the front and back flippers, the net moment is:

$$
M=\left(2 d_{f}+2 d_{b}\right)\left(F_{l} \cos \theta+F_{t} \sin \theta\right)
$$

Expanding $F_{l}$, we get:

$$
M=\left(2 d_{f}+2 d_{b}\right)\left\{\left(K_{a} \sin \theta+K_{b} \dot{\theta}^{2}\right) \cos \theta+F_{t} \sin \theta\right\}
$$

with $\sin \theta=\theta$ and $\cos \theta=1$, (8) simplifies to:

$$
\left.M \approx\left(2 d_{f}+2 d_{b}\right)\left\{\left(K_{a}+F_{t}\right) \theta+K_{b} \dot{\theta}^{2}\right)\right\}
$$

Approximating the moment arms $d_{f}$ and $d_{b}$ as constant, we can group the constants together, thus (9) simply becomes

$$
M \approx K_{1} \theta+K_{2} \dot{\theta}^{2}
$$

This shows that the moment generated by the flippers can be approximated by a two-constant equation. The $K_{1}$ parameter represents the combined moments generated by the oriented thrusters and surface lift, whereas $K_{2}$ captures the transient moments due to the moving surface. 


\section{Added Model Features}

To be able to have a better match, some extra parameters have been added to the model:

\section{1) Motor torque saturation $T_{\text {sat }}$}

With the squared factor of the derivative in the torque calculation, spikes rapidly appear in the torque generation. Given the limited motor characteristics, not limiting the torque in the model would make the robot react ahead of time.

\section{2) Moment offset $M_{\text {off }}$}

A moment offset could be added to simulate twisted flippers or external disturbances such as tether drag forces or currents from the pool filter jets.

\section{Complete Model}

Fig. 4 shows final model used in the matching the response for each axis.

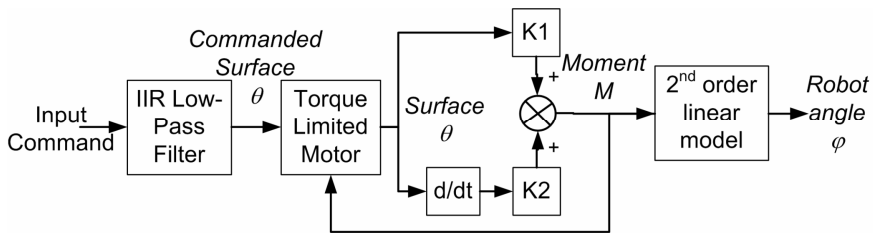

Fig. 4. Single-axis model used for end-to-end simulation of the robot. The collected input command is fed into the model, and the output robot angle is

$\varphi$. The low-pass filter is used in the real system to smooth the input command in order to avoid abrupt surface changes that would put large stresses on the flippers. The moment $\mathrm{M}$ feedback is needed to simulate the torque limitation of the motor.

The model parameters $I, C, K_{1}, K_{2}, T_{\text {sat }}$ and $M_{\text {off }}$ were manually tuned to give a good qualitative match for a series of test cases covering all three axes.

\section{E. Pitch Axis Results}

Fig. 5, 6 and 7 show the comparison between the actual robot pitch angles and the tuned model output for commands of $100 \%, 50 \%$ and $25 \%$ of maximum. The quality of the match is excellent for the $100 \%$ and $50 \%$ command. In these cases, the match is within $3^{\circ}$ (less than $5 \%$ error compared to the full test range) for the sequence used to tune the model, and less than $8^{\circ}$ for the validation test case. For the $50 \%$ test case, the damping factor had to be reduced by about $30 \%$, which was expected since the true nature of water damping is more squared than proportional. a)
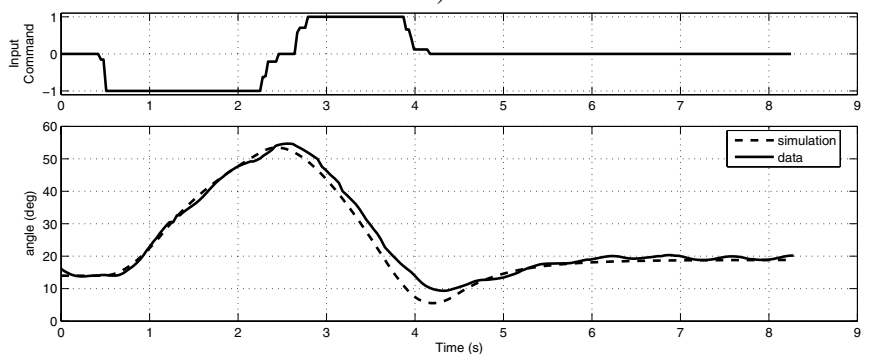

b)
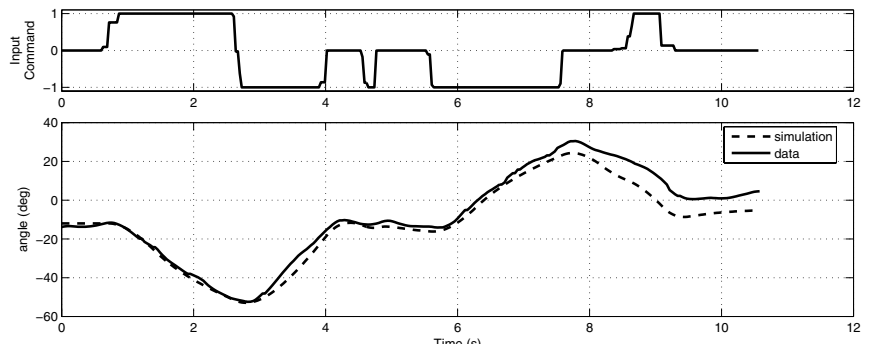

Fig. 5. Model and vehicle responses for pitch commands of $+/-100 \%$. a) shows the data used to tune the model, and b) is the response for a different test using the same parameters as in a).

a)
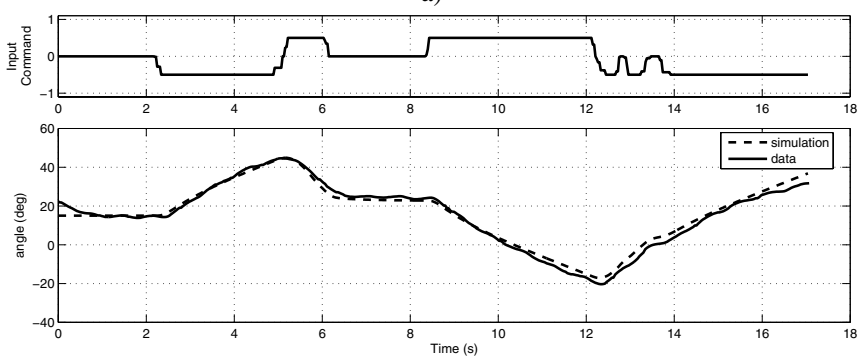

b)
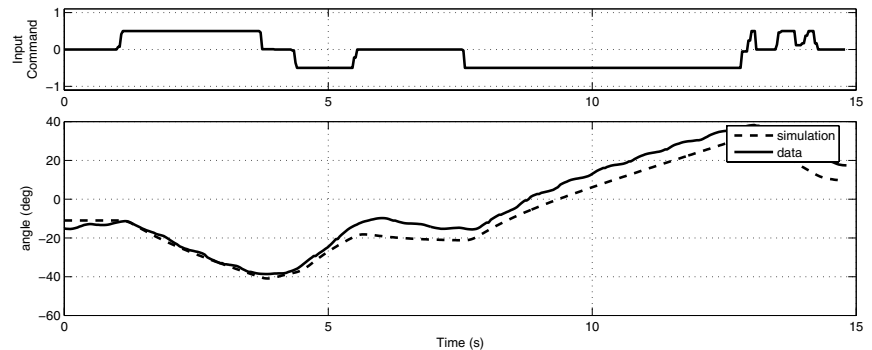

Fig. 6. Model and vehicule response for a series of pitch command of $+/$ $50 \%$. a) is the test sequence used for tuning the parameters, and b) is the response for a different test input using the same parameters as in a).

For the $25 \%$ case, the flipper parameters $K_{I}$ had to be decreased by $15 \%$ and $K_{2}$ by $50 \%$. These could be the result of non-linear phenomenon more apparent at small commands, such as a dead-band effect around the neutral position due to flexibility of the surface.

\section{E. Roll Axis Results}

Fig. 8 shows the results for a full negative roll command ($100 \%)$. Notice that the duration of the test is much shorter than the other cases, giving the appearance of a not-so-good match. But looking closely at the Figure, the model delay prediction is only off by $150 \mathrm{~ms}$. This is probably due to an 
overestimation of motor torque saturation, one of the major components of delay in the model. However the free response decay from time $2.0 \mathrm{~s}$ to $3.1 \mathrm{~s}$ is a dead-on match.

a)

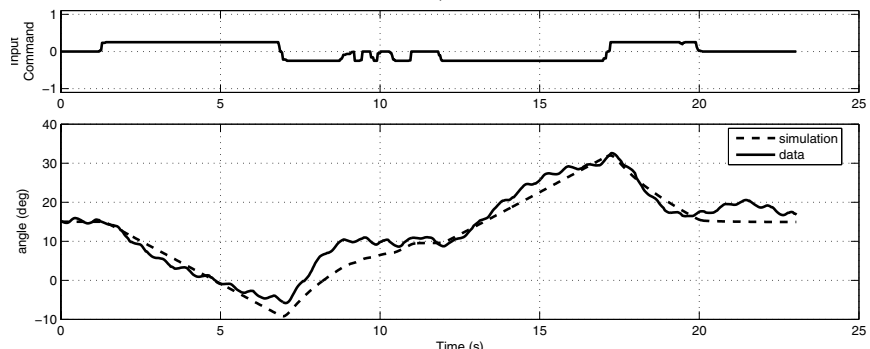

b)

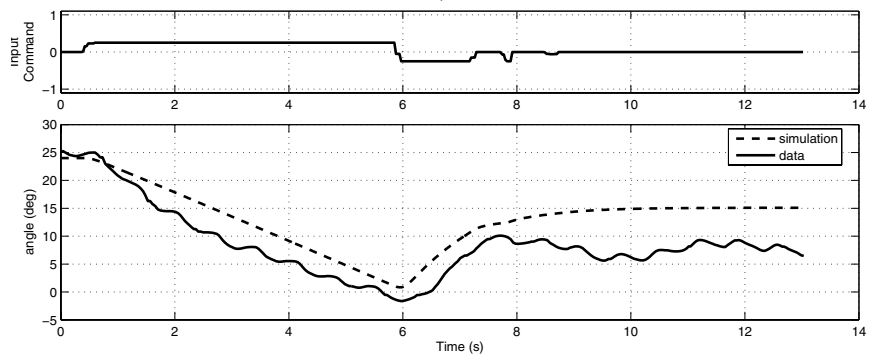

Fig. 7. Model and vehicle responses for a series of pitch command of $+/$ $25 \%$. a) is the test sequence used for tuning the parameters, and b) is the response for a different test input using the same parameters as in a).

a)

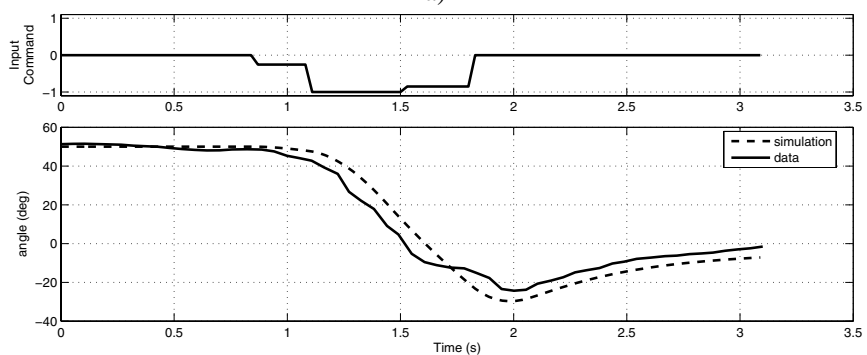

b)

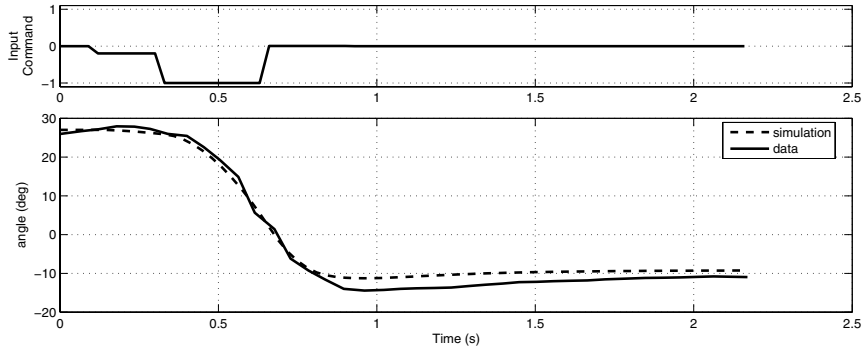

Fig. 8. Model and vehicle responses for a roll command of $-100 \%$. a) is the test sequence used for tuning the parameters, and $b$ ) is the response for $a$ different test input using the same parameters as in a).

Fig. 9 shows the results for a combination of $+/-25 \%$ roll commands. One noticeable feature of the vehicle response is the sharp changes of roll angular rate, indicating that the vehicle has a near-ideal response at this command level. This fast response is the result of the transient forces. This validates our choice of time constant for the command filtering, telling us that a larger value would be detrimental to the vehicle responsiveness. The change in positive angle slopes (first one between $2 \mathrm{~s}$ and $7 \mathrm{~s}$, the second between $18 \mathrm{~s}$ and $25 \mathrm{~s}$ ) clearly indicates that there is some limit in the reproducibility of the response of the vehicle. At this moment we have no explanation for this.

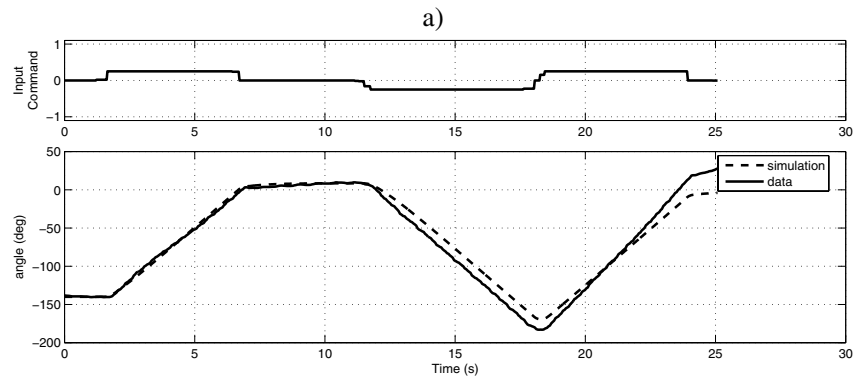

b)
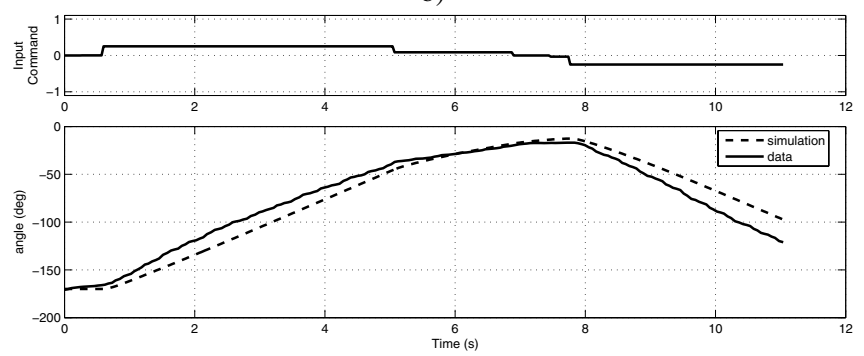

Fig. 9. Model and vehicle responses for a roll command of $+/-25 \%$. a) is the test sequence used for tuning the parameters, and $b$ ) is the response for $a$ different test input using the same parameters as in a).

a)
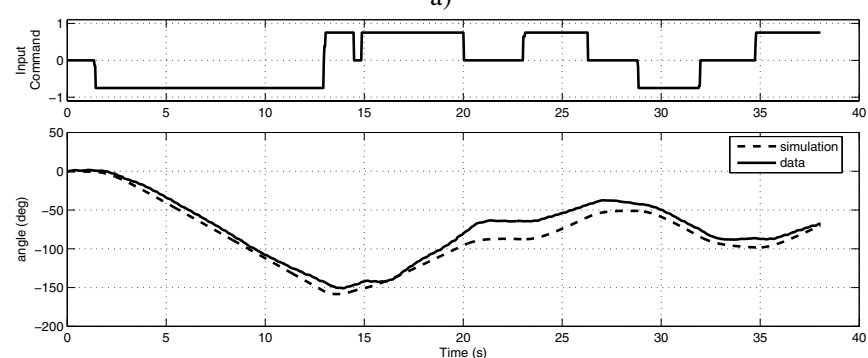

b)
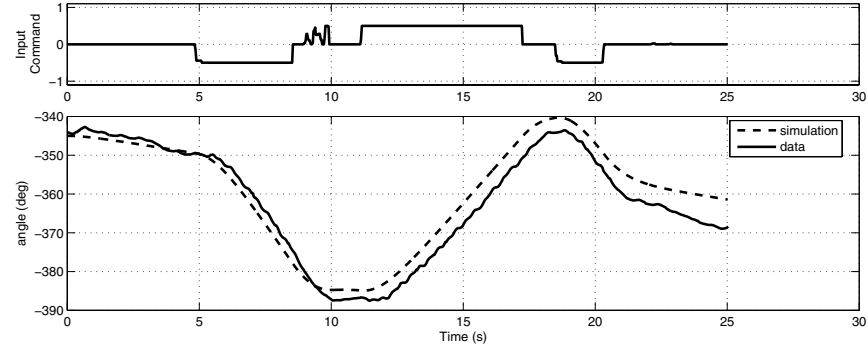

Fig. 10. Model and vehicle responses for a yaw command of a) $+/-75 \%$ and b) $+-50 \%$ The surface corresponds to a virtual surface acting to generate the yawing moments.

\section{F. Yaw Axis Results}

The same model is used for the yaw axis, even though the underlying mechanism is quite different (difference of thrust vs. lift forces). A significant difference is that the transient forces term $K_{2}$ will be very small compared to the other axes. By looking at the physics of the system, one would conclude the same, since no surface is being deployed. The fact that the term is smaller also helps explain the much greater delay associated with the yaw axis. 
Although the fit of the model for the yaw axis is not quite as good as for pitch and roll, the model still captures the qualitative behavior of the robot and is an acceptable fit to the data for our navigation applications (Fig. 10). The delay in command execution is easily captured by the model, as are the angular rates. A small moment offset $(-0.03)$ was added to get a better match. This offset is consistent with the values seen in the steady-state rates showed in Fig. 3.

\section{CONCLUSION}

We have demonstrated that using a simple two-layer empirically-based system model allows us to accurately predict the behavior of our swimming robot. Prior work based on an analytic model of the vehicle hydrodynamics, while elegant and effective, was less accurate as on overall performance predictor. While model tuning is a costly procedure, requiring the collection of empirical data, the total time required is comparable that that needed to develop and tune an analytic model.

Using oscillating foils for propulsion and control enables our underwater vehicle to be highly maneuverable. In our design, the pitch and roll axis benefits largely from the large transient forces generated by surface drag. The command delay for those axes were less than $300 \mathrm{~ms}$. On the other hand, the yaw axis response is much slower $(1300 \mathrm{~ms})$, due in great part to a lack of those forces.

Even though the vehicle dynamics are quite complex, we showed that in the case of this particular robot, each axis can be approximated with a simple model with 5 parameters. This simplified model arises when a number of simplifications are assumed. The reasonable match between the tuned model and the experimental data validates the simplifications used to derive the model. A positive aspect of this simple model is the straightforward hindsight it gives about the vehicle dynamics.

However limitations in the linear modeling of the vehicle dynamics are apparent. In particular, it partially fails to properly capture the damping forces of the vehicle body in the water. This can be seen in the variation in the damping factor $C$ for different test cases for identical axis. Initial testing with a non-linear model indicates that a mixture of squared and proportional damping would provide a closer match to the data seen across all body angular velocities.

To improve matches, a more complex model for moment generation could also be developed. This would take into account the interactions between the vehicle motion and the moments generated, since drag and lift are dependent on the incoming water speed. This might explain the use of various torque limits for pitch and roll, since the body starts rotation much faster in the roll case. With the current model also, the moments are symmetric for surface extensions and retraction. There seems to be indication that in fact the forces are asymmetric, with surface extension from the neutral angle generating more forces than retraction.

\section{ACKNOWLEDGMENT}

P. G. thanks Shane Saunderson from the MLL lab at McGill University for his immense help and assistance during this data-gathering project. P.G. would thanks as well Junaed Sattar, Paul DiMarco, Daniel Burfoot and Dave Meager from the MRL lab for helping out during the actual experiments.

\section{REFERENCES}

[1] M. Nahon, "A simplified dynamics model for autonomous underwater vehicles”, Proc 1996 Symp. on Autonomous Underwater Vehicle Technology, New York, NY, IEEE, 373-379, 1996.

[2] K. R. Goheen, "Modeling Methods for Underwater Robotic Vehicle Dynamics", Journal of Robotic Systems, Vol. 8, No 3, pp 295-317, 1991.

[3] C. Georgiadis et al., "AQUA: An Aquatic Walking Robot", IEEE/RSJ International Conference on Intelligent Robots and Systems, IROS 2004, Sendai, Japan, September 28 - October 2, 2004

[4] R. Altendorfer et al., "Rhex: A Biologically Inspired Hexapod Runner", Autonomous Robots 11:207-213, 2001

[5] J. Katz, A. Plotkin, "Low Speed Aerodynamics: From Wing Theory to Panel Methods", McGraw-Hill, New York, 1991.

[6] S.N. Singh, A. Simha, R Mittal, "Biorobotic AUV Maneuvering by Pectoral Fins: Inverse Control Design Based on CFD Parameterization , IEEE Journal of Oceanic Engineering, Vol. 29, No.3, 777-785, July 2004.

[7] D.Barrett, M.Grosenbaugh and M.Triantafyllou, "The optimal control of a flexible hull robotic undersea vehicle propelled by an oscillating foil", Proceedings of the IEEE Symposium on Autonomous Underwater Vehicle Technology, 1-9, 1996.

[8] B. Hobson, M. Kemp, A. Leonessa, "Integration of a hovering module with the Morpheus AUV: application to MCM missions", MTS/IEEE Oceans, Volume 1, 29-31, pp. 207 - 209, Oct. 2002.

[9] M. Kemp, B. Hobson, J. Janet, C. Pell and E. Tytell, "Assessing the Peroformance of Oscillating Fin Thruster Vehicles", Proceedings of 12th International Symposium on Unmanned, Untethered Submersible Technology, 2001.

[10] Hobson, B. Murray, M. and Pell, C. "Pilotfish: Maximizing Agility in an Unmanned Underwater Vehicle" (pg. 41-51), Int Symp on Unmanned Untethered Submersible Tech. 1999. 